\title{
National Survey of Lymphedema Therapists' Dosing of Complete Decongestive Therapy in Breast Cancer Survivors with Lymphedema
}

\author{
Katie M. Polo \\ University of Indianapolis, polok@uindy.edu \\ Peter J. Rundquist \\ Concordia University, rundquist@csp.edu \\ Nathaniel D. Krumdick \\ Midwestern University, nkrumd@midwestern.edu \\ Gail L. Gamble \\ Northwestern University, ggamble33@gmail.com
}

Follow this and additional works at: https://nsuworks.nova.edu/ijahsp

Part of the Occupational Therapy Commons, and the Physical Therapy Commons

\section{Recommended Citation}

Polo KM, Rundquist PJ, Krumdick ND, Gamble GL. National Survey of Lymphedema Therapists' Dosing of Complete Decongestive Therapy in Breast Cancer Survivors with Lymphedema. The Internet Journal of Allied Health Sciences and Practice. 2018 Jan 01;16(1), Article 5.

This Manuscript is brought to you for free and open access by the College of Health Care Sciences at NSUWorks. It has been accepted for inclusion in Internet Journal of Allied Health Sciences and Practice by an authorized editor of NSUWorks. For more information, please contact nsuworks@nova.edu. 


\title{
National Survey of Lymphedema Therapists' Dosing of Complete Decongestive Therapy in Breast Cancer Survivors with Lymphedema
}

\begin{abstract}
Purpose: The purpose of this study was to identify dosing levels of complete decongestive therapy (CDT) with survivors with Breast Cancer-Related Lymphedema (BCRL) and describe factors related to how occupational therapists, occupational therapy assistants, physical therapists, and physical therapists assistants with advanced lymphedema training determine dosing.

Methods: An electronic survey was sent to 598 occupational therapists, occupational therapy assistants, physical therapists, and physical therapists assistants with training in lymphedema. Respondents completed questions regarding demographics, dosing practices, and factors related to dose determination in complete decongestive therapy of survivors with Breast Cancer-Related Lymphedema.

Results: 107 surveys were returned (18\% response rate). Most of the respondents $(95.1 \%)$ reported providing less than the recommended daily dosing $(7 \mathrm{x} / \mathrm{wk})$ of complete decongestive therapy to their survivors with Breast Cancer-Related Lymphedema. The mean dosing of complete decongestive therapy from respondents was approximately half of the recommended daily dosing $(M=3.71)$. A statistically significant Pearson's correlation was noted among frequency of dosing and treatment adherence $(r=.275)$ and frequency of dosing and treatment readiness ( $r=.242)$. A multiple regression analysis found adherence accounted for a significant proportion of variability in dosing frequency $\left(R^{2}=.077 ; F\right.$ change $\mathrm{p}$

Conclusions: A majority of therapists treating survivors with Breast Cancer-Related Lymphedema in an outpatient setting are not following daily recommended dosing of complete decongestive therapy. Clinicians' dose determination is significantly impacted by their perception of a survivor's readiness and adherence to lymphedema treatment and self-management. Comparative clinical outcomes studies of various therapeutic dosage levels (times per week) and duration (length of delivery) of complete decongestive therapy are imperative for development of best treatment protocols for survivors with Breast Cancer-Related Lymphedema. This study serves as a first step toward evidence based planning for complete decongestive therapy treatment of Breast Cancer-Related Lymphedema.
\end{abstract}

\section{Author Bio(s)}

Katie M. Polo, DHS, OTR, CLT-LANA is an Assistant Professor, School of Occupational Therapy, College of Health Sciences, University of Indianapolis in Indianapolis, IN.

Peter J. Rundquist, PT, PhD is Director, Doctor of Physical Therapy Program, Concordia University in Saint Paul, MN.

Nathaniel D. Krumdick, Ph.D. is Associate Professor, Department of Clinical Psychology, Midwestern University, Downers Grove, IL.

Gail L. Gamble, M.D. is Emeritus Professor, Feinberg School of Medicine, Northwestern University, Chicago, IL. 


\title{
IJAHSP \\ The Internet Joumnal of Allied Health Sciences and Practice \\ Dedicated to allied health professional practice and education
}

Vol. 16 No. 1 ISSN 1540-580X

\section{National Survey of Lymphedema Therapists' Dosing of Complete Decongestive Therapy in Breast Cancer Survivors with Lymphedema}

\author{
Katie M. Polo, DHS, OTR, CLT-LANA 1 \\ Peter J. Rundquist, $\mathrm{PT}, \mathrm{PhD}^{2}$ \\ Nathaniel D. Krumdick, Ph.D. ${ }^{3}$ \\ Gail L. Gamble, M.D. ${ }^{4}$ \\ 1. University of Indianapolis \\ 2. Concordia University \\ 3. Midwestern University \\ 4. Northwestern University
}

United States

\begin{abstract}
Purpose: The purpose of this study was to identify dosing levels of complete decongestive therapy (CDT) with survivors with breast cancer-related lymphedema (BCRL) and describe factors related to how occupational therapists, occupational therapy assistants, physical therapists, and physical therapist assistants with advanced lymphedema training determine dosing. Methods: An electronic survey was sent to 598 occupational therapists, occupational therapy assistants, physical therapists, and physical therapist assistants with training in lymphedema. Respondents completed questions regarding demographics, dosing practices, and factors related to dose determination in complete decongestive therapy of survivors with BCRL. Results: 107 surveys were returned (18\% response rate). Most of the respondents $(95.1 \%)$ reported providing less than the recommended daily dosing (7x/wk) of complete decongestive therapy to their survivors with BCRL. The mean dosing of complete decongestive therapy from respondents was approximately half of the recommended daily dosing $(M=3.71)$. A statistically significant Pearson's correlation was noted among frequency of dosing and treatment adherence $(r=.275)$ and frequency of dosing and treatment readiness $(r=.242)$. A multiple regression analysis showed adherence accounted for a significant proportion of variability in dosing frequency $\left(R^{2}=.077, F\right.$ change $\left.p<.05\right)$. Conclusions: A majority of therapists treating survivors with $B C R L$ in an outpatient setting are not following daily recommended dosing of complete decongestive therapy. Clinicians' dose determination is significantly affected by their perception of a survivor's readiness and adherence to lymphedema treatment and self-management. Comparative clinical outcomes studies of various therapeutic dosage levels (times per week) and duration (length of delivery) of complete decongestive therapy are imperative for development of best treatment protocols for survivors with BCRL. This study serves as a first step toward evidence-based planning for complete decongestive therapy treatment of BCRL.
\end{abstract}

\section{BACKGROUND}

A woman in the United States has about a 1 in 8 chance of developing breast cancer in her lifetime, and men with breast cancer gene (BRCA1 or BRCA2) have a 1 in 100 or 6 in 100 chance, respectively, of developing breast cancer in their lifetimes. ${ }^{1}, 2$ Even more alarming is that many of the 2.8 million breast cancer survivors living in the United States today are living with lymphedema, a chronic non-curable side effect of breast cancer treatment. ${ }^{3}$ Breast cancer-related lymphedema (BCRL) is an abnormal accumulation of protein-rich fluid in the interstitial spaces that can lead to chronic inflammation of tissues; swelling of the limb, shoulder girdle, breast, or thoracic region; or a combination of any of these. ${ }^{4}$ Chronic lymphedema can lead to impairment in upper extremity function, ultimately affecting a survivor's daily occupational performance in activities of daily living (ADL). ${ }^{5}$ Regardless of the stage, severity, or etiology, most survivors are treated similarly with complete decongestive therapy (CDT), which has been found to likely be effective in a systematic review of the literature. ${ }^{6,7}$ 
Traditional complete decongestive therapy for chronic lymphedema consists of two phases: an intensive phase (phase I), performed by a therapist with advanced lymphedema training and a maintenance phase (phase II) that transitions the patient to self-management of lymphedema at home. The intensive phase has a recommended standardized protocol, focusing on meticulous skin and nail care, manual lymphatic drainage, compression bandaging, and remedial exercises, and is recommended to be dosed daily by a trained therapist until maximal volume reduction and normalization of tissue texture is achieved. ${ }^{8}$ After a review of literature in dosing of CDT, study protocols varied significantly between both number of days per week and overall duration of treatment provided. For the purposes of this study, dosing in lymphedema management is the frequency of treatment (times per week) as opposed to overall time (minutes of treatment) and duration of treatment.

Due to multiple limitations of the United States health care system, including reimbursement of therapy services, the number of out-of-pocket patient expenses, and caps on therapy treatment, therapists with advanced lymphedema training likely are struggling to provide the recommended daily outpatient dosing of intensive phase I CDT to survivors. An investigation of the daily lymphedema reduction patterns during the CDT intensive phase showed the most substantial lymphedema reduction occurs within the first 3 days of treatment. ${ }^{9}$ Similarly, in two studies, the most significant reduction in limb volume was achieved during the first week of treatment. ${ }^{10,11}$ These results have been used to support shortening the CDT intensive treatment phase to reduce economic burden of the patients.

Although CDT is supported by efficacy studies, the daily dosing recommendation was developed using level five evidence and is anecdotal rather than evidence-based practice. Knowledge of potential factors that influence CDT outcomes could assist clinicians in developing plans of care and need to be further explored. ${ }^{7}$ The wide range of selected dosing of CDT in studies and the lack of comparative dosing within studies demonstrates a need for effective protocol development for best survivorship care. Studies for comparing dosing for effective CDT protocols and factors influencing dose determination have not yet been performed. Therefore, research is necessary to determine the current state of CDT practice and factors that affect dose determination in the United States.

In the literature, CDT has varied dosing from 1 time per week to 7 times per week; therefore, research efforts are indicated to explore the state of CDT practice today and factors associated with dose determination, which will allow for a better understanding of current practice and the potential need for further efficacy studies for dose comparison. The primary purpose of this study was to identify lymphedema trained therapists' dosing levels (times per week) of phase I CDT with survivors with BCRL. The secondary purpose was to describe factors related to how therapists determine dosing of phase I CDT for survivors with BCRL. Specifically, the frequency (times per week) of phase I CDT dosing provided by lymphedema therapists is described in this study, and the organizational and client factors related to CDT dose determination in survivorship have been identified.

\section{METHODS}

\section{Research Design}

A survey was developed for occupational therapists, occupational therapy assistants, physical therapists, and physical therapist assistants with advanced training in lymphedema that assessed participant demographics, dosing practices, and factors related to dose determination in phase I treatment using CDT for survivors with BCRL. The survey consisted of four sections assessing current practice on dosing of $\mathrm{CDT}$ and factors related to dose determination. A combination of questions that used fixed response options, Likert scales, and free text comments were used in the design: (1) Demographic information (6 items of fixed response options and free text comments), (2) current CDT dosing practice (three items of fixed response options and free text comments), (3) perceptions on client factors that inhibit provision of intended plan of care (10 Likert-scale items), and (4) perceptions about organizational factors that inhibit provision of intended plan of care (4 Likert-scale items).

\section{Survey Instrument Development}

To assure the survey design met the needs of the respondents and to check face validity, a pretest of the survey was performed before dissemination. This pretest was used for researchers to identify potential problems with intent, clarity, and navigation..$^{12}$ The pretesting process for developing the questionnaire was conducted using recommended steps. ${ }^{13}$ Five certified lymphedema therapists (2 occupational therapists and 3 physical therapists) working with survivors with BCRL were given the survey via paper and contacted for a telephone interview. The therapists were asked to first answer the questions just as if they were research participants, then conversation was opened to critiques of the preliminary version of the survey. Information gleaned from these pretests was incorporated into minor changes for the final survey, and changes were approved

(C) The Internet Journal of Allied Health Sciences and Practice, 2018 
by these experts. This study was approved and conducted in accordance with the University of Indianapolis's institutional review board.

\section{Participants}

Nonprobability and snowball sampling approaches were utilized through the first investigator's professional networks, including personal network of colleagues, the National Lymphedema Network conference attendee list, and the American Occupational Therapy Association's professional networking site. Those therapists with a background in physical or occupational therapy with advanced training in lymphedema were asked to participate.

\section{Procedures}

REDCap software Version 5.8.2, an online survey development and data collection secure Web application, was utilized. ${ }^{14}$ Therapists were emailed an invitation to participate in the study, and a direct link to the online survey was included. Informed consent was provided as the front page of the survey, and participants' submission indicated their consent. To address nonresponse error, surveys were distributed to possible participants twice as a reminder strategy. ${ }^{12}$ The survey results were analyzed using IBM SPSS Version 22 (IBM Corporation, Armonk, NY). The data were analyzed using descriptive statistics and a series of correlation and regression analyses to explore the research questions.

\section{RESULTS}

\section{Respondents}

Six hundred forty-four practitioners were initially invited to complete the survey; 46 surveys were returned as undeliverable, resulting in 598 potential participants. One hundred and seven responded to the survey for a response rate of $18 \%$. All respondents but 4 were female with an average age of approximately 46 years old $(M=45.78, S D \pm 9.720)$. Most of the respondents $(81.1 \%, n=90)$ reported having a master's degree or lower for their educational background. The average number of years respondents practiced was almost 9 years $(M=8.94, S D \pm 2.927)$. Table 1 presents respondents' demographics and further professional characteristics.

Table 1. Respondent Demographics, Credentials, Educational Background, and Years of Practice

\begin{tabular}{|l|l|l|}
\hline Demographics, Credentials, \& Years of Practice & \multicolumn{1}{|c|}{$n$} & Valid Percent \\
\hline Gender & \multicolumn{1}{|c|}{$\mathbf{n}$} & 4.4 \\
\hline Male & 4 & 95.6 \\
\hline Female & 86 & \\
\hline Ethnicity & & 92.2 \\
\hline Caucasian & 83 & 4.4 \\
\hline African-American & 4 & 0 \\
\hline Hispanic & 0 & 2.2 \\
\hline Asian & 2 & 1.1 \\
\hline Pacific Islander & 1 & \\
\hline Credentials & & 1.1 \\
\hline Occupational Therapy Assistant & 1 & 36.7 \\
\hline Occupational Therapist & 33 & 6.7 \\
\hline Physical Therapist Assistant & 6 & 50.0 \\
\hline Physical Therapist & 45 & 65.6 \\
\hline Certified Lymphedema Therapist & 59 & 56.7 \\
\hline LANA Certified Therapist & 51 & \\
\hline Educational Background & & 1.1 \\
\hline Associate's Degree & 1 & 43.3 \\
\hline Bachelor's Degree & 39 & 35.6 \\
\hline Master's Degree & 32 & 18.9 \\
\hline Clinical Doctorate & 17 & $S D$ \\
\hline & $M$ & 9.720 \\
\hline Age & 45.78 & 2.927 \\
\hline Year of Practice & 8.94 & \\
\hline & & \\
\hline
\end{tabular}




\section{Response to Dose Provision}

Most of the respondents (95.1\%) reported providing less than the recommended daily dosing (7x/wk) of CDT to their patients with BCRL. Specifically, the mean dosing of complete decongestive therapy was 3.71 times per week with a standard deviation of 1.35 . Figure 1 depicts the group frequency distribution related to dose provision.

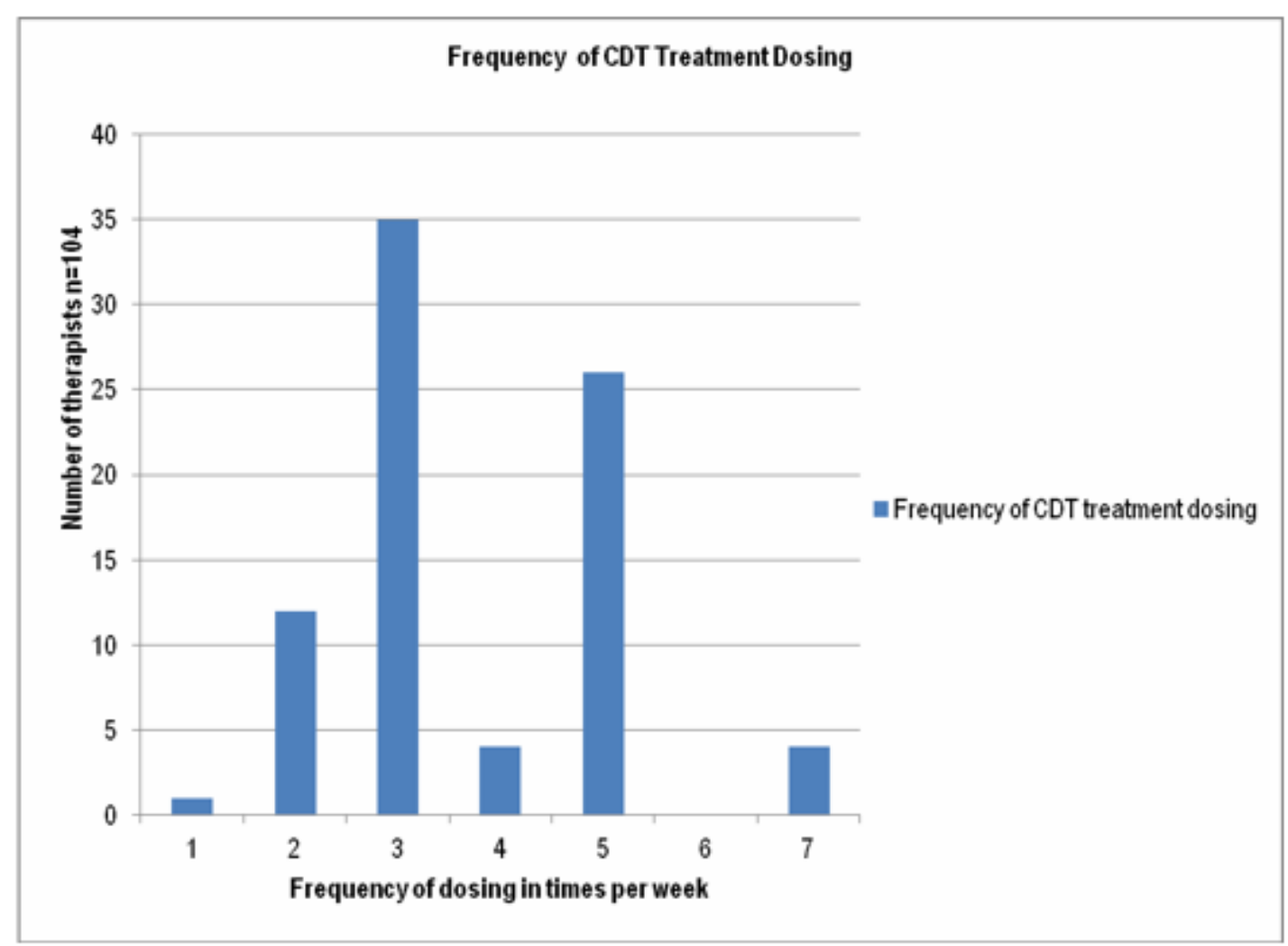

Figure 1. Histogram of Mean and Standard Deviation of Treatment Dosing

\section{Perceived Factors Affecting Dose Determination}

The top perceived factors indicated by respondents as most affecting CDT dose determination included the clients' schedule $(M=8.03, S D \pm 2.06)$, client's transportation availability $(M=7.90, S D \pm 2.33)$, and client's readiness for treatment $(M=7.50$, $S D \pm 2.58$ ). The factors indicated by respondents as having the least effect on CDT dose determination include not enough lymphedema trained clinicians $(M=3.91, S D=3.55)$, organizational policies $(M=3.50, S D \pm 3.58)$, and organizational culture $(M=2.92, S D \pm 3.37)$. Table 2 depicts the mean responses and standard deviation related to perceived factors affecting CDT dose determination.

\section{Correlation of Perceived Factors Related to CDT Dosing}

A statistically significant Pearson's correlation was noted among frequency of dosing and treatment adherence $(r=.275)$ and treatment readiness $(r=.242)$. Table 2 depicts the correlations of each of the perceived factors affecting CDT dosing.

\section{Regression of Perceived Factors Related to CDT Dosing}

A forward, stepwise, multiple regression analysis was performed to clarify the relationships between the two correlated perceived factors and frequency of dosing. The regression analyses only included outpatient therapists because a disproportionate respondent distribution was noted within therapists practice setting with significantly more respondents from an outpatient setting. By itself, adherence accounted for a significant proportion of the variability in dosing frequency $\left(R^{2}=\right.$ $.077, F$ change $P=.042$ ). However, adding readiness to this predictive model did not improve the overall amount of variability explained (Adjusted $R^{2}=.054 ; F$ change $=.042, F$ change $p=.717$ ). Thus, it appears that the significant correlation between 
readiness and dosing maybe due to an underlying correlation between readiness and adherence. Table 2 depicts the correlation regression statistics.

Table 2. Mean, Standard Deviation, and Pearson Correlation of Perceived Factors Affecting Dose Determination

\begin{tabular}{|l|r|l|r|}
\hline Factor & M & SD & Correlation (r) \\
\hline Volume in UE & 7.40 & 3.008 & .095 \\
\hline Symptoms & 7.00 & 2.879 & .176 \\
\hline Other Diagnoses & 5.92 & 2.785 & .160 \\
\hline Patient Supports & 7.46 & 2.442 & .187 \\
\hline Patient Schedule & 8.03 & 2.064 &. .008 \\
\hline Availability of Transportation & 7.90 & 2.326 & .124 \\
\hline Patient Readiness & 7.50 & 2.580 & $.242^{\star}$ \\
\hline Patient Adherence & 7.37 & 2.486 & $.275^{\star}$ \\
\hline Insurance & 6.26 & 3.616 & .088 \\
\hline Patient Finances & 6.50 & 3.226 & -.031 \\
\hline Therapists Availability & 5.30 & 3.790 & .033 \\
\hline Organizational Culture & 2.92 & 3.370 & -.123 \\
\hline Organizational Policies & 3.50 & 3.584 & -.051 \\
\hline Staffing & 3.91 & 3.549 & -.118 \\
\hline
\end{tabular}

Correlation is significant at the 0.05 level (2-tailed).

*Correlation is significant at the 0.01 level (2-tailed)..*

\section{DISCUSSION}

The primary purpose of this study was to identify lymphedema trained therapists' provision of dosing levels (times per week) of CDT for patients with BCRL. While traditional protocols call for intensive phase I complete decongestive therapy (CDT) that includes daily dosing, this study's findings indicated that around $95 \%$ of therapists are not providing this recommended protocol in the United States. The average rate of dosing reported was just over half the recommended dosing at 3.71 days per week. When ranges in frequency of dosing were reported by respondents, the highest value reported was used with the assumption that a range reflects the fact that this treatment is intended to taper overtime. Respondents' frequency of CDT dose delivery ranged from 1 time per week to 7 times per week.

It is still unknown whether the dosing found in this study is the appropriate amount to create maximum therapeutic benefit, thus further studies are needed comparing the effectiveness of different frequencies of dosing (times per week) and duration of dosing (length of delivery) for the provision of the most evidence-based protocol recommendations. This recommendation was supported by several researchers in concluding that longer-term follow-up studies are needed to determine optimal treatment protocols of CDT. $7,15,16$ Holtgrefe found that twice weekly dosing with a higher dosing frequency similar to 5 days per week is needed. ${ }^{17}$ This study showed that phase I CDT dosing varies so widely that future studies will also need to explore other dosing variables beyond 2 and 5 times a week. It has also been recommended by Yamamoto et al that for future randomized controlled trials, researchers should consider reducing phase I of CDT and also consider therapeutic effects to optimize use of limited financial resources. ${ }^{9}$

Physical therapist assistants (PTA) were included as participants because they may perform the dosing recommended by their supervising physical therapist. Of important note, this study was conducted under the assumption that therapists were adhering to all components of phase I CDT practice (skin and nail care, manual lymphatic drainage, compression bandaging, remedial exercises, and self-care training). Researchers have found that lymphedema treatment itself (components of treatment, such as manual lymphatic drainage, bandaging, and garments) vary significantly. ${ }^{18}$ This study was not focused on treatment content nor specific clinical outcomes for the patients. Thus, research is also needed to study individual components of treatment along with differing combinations of treatments in order to more accurately develop optimal treatment protocol recommendations.

The secondary purpose of this study was to determine factors related to how therapists with advanced lymphedema training 
determine dosing of $\mathrm{CDT}$ for survivors with BCRL. Top factors perceived by therapists as affecting their dosing were all client factors: availability of transportation, patients' schedule, and patient readiness. The factors that were perceived by therapists as least influential in dose determination were all organizational factors: staffing, organizational policies, and organizational culture. Thus, organizational barriers are not perceived as affecting dose determination in lymphedema survivorship care. The distribution of these factors was slightly skewed, which may be a function of the Likert scale used in assessment.

It is not surprising that our study indicated a disproportionate respondent distribution because generally the United States health care system model is not supportive of inpatient lymphedema treatment. Statistically significant correlations provided evidence that a survivor's readiness and adherence to treatment affect therapists' dose determination. Patients with chronic diseases such as BCRL must possess a readiness for management and treatment of their condition or disease progression and non-adherence can be consequences. ${ }^{20}$ Both adherence and readiness factors are imperative for self-management of chronic conditions such as lymphedema. The regression analysis showed adherence to account for approximately $8 \%$ of the predictive model. Thus, continued studies are needed to further explore additional factors that might explain the remaining variation in therapists' dose determination. Reserchers should add a survey validation process into their methods and focus on patients' perceptions of dose determination to potentially account for more of the predictive model.

Lymphedema therapists who perceived self-management factors of adherence and readiness as issues affecting provision of CDT dosing more often selected a higher frequency of CDT dosing to their patients. Self-management ultimately is imperative to reduce exacerbations of swelling, prevent infection, and manage other symptoms associated with lymphedema. ${ }^{21}$ Thus, it is not surprising that the higher the therapists scored on the self-perceived factors of self-management, the higher the dosing they provide to their patients. Although clinicians are indicating that self-management factors influence the dosing of CDT they provide, there is little evidence on which to make recommendations for self-management. ${ }^{21}$ Further studies are warranted to explore patient's perceptions of dosing factors. Patient perspectives on which factors are most important to support selfmanagement strategies should also be explored.

This study indicated that clinicians are considering self-management factors, such as readiness and adherence to treatment, which affect dose determination in phase I of CDT. The average adherence to BCRL self-care is not adequate. ${ }^{22}$ Additional researchers have recommended further exploration of the role of patients' adherence to maintenance of their selfmanagement programs. ${ }^{7,19}$ Therapists working with survivors should consider each patient's lymphedema self-management needs alongside provision of resources to inform and facilitate the development of an individualized self-management plan. 19

\section{Study Limitations}

This study has several limitations based on the utilization of the nonprobability sampling approach. Primarily, the sample was limited to the National Lymphedema Network conference attendee list and other nonprobability sampling; therefore, it may not be representative of all therapists with advanced training in lymphedema practicing within the United States. This study's response rate, however, did fall between recommended liberal and stringent conditions required for survey response rates. ${ }^{23}$ The participants were all therapists providing treatment to survivors with BCRL, thus the respondents might have had an elevated interest in the topic. Additionally, this study can only provide the therapists' perspectives, which may differ from the survivors.

Although the survey instrument was developed after a thorough literature review and feedback was incorporated from pretesting the instrument with experts in the field, a formal validation process was not completed. The use of closed-ended questions and a Likert scale (range of 0-10) with a ceiling may have limited the scope of participant's responses.

\section{CONCLUSION}

This study indicated that a majority of practicing occupational therapists, occupational therapy assistants, physical therapists, and physical therapist assistants with advanced lymphedema training in outpatient settings in the United States are not following daily recommended dosing in phase I CDT. Additionally, clinicians' dose determination is significantly affected by their perception of a survivor's external supports, readiness, adherence to lymphedema treatment and self-management of this chronic condition. Practicing clinicians with advanced lymphedema training need to be mindful of the dosing they provide survivors with BCRL and continue to individually tailor dosing based on patient needs until further research explorations provide evidence for guidance. Future research is recommended to determine the most effective dosing for phase I CDT to improve survivorship outcomes. Furthermore, researchers should explore survivor's readiness for treatment and its effect on adherence

(c) The Internet Journal of Allied Health Sciences and Practice, 2018 
to treatment as well as overall lymphedema self-management strategies for a more holistic approach to this chronic disease. Future researchers have the potential to determine if clinicians need to consider initially monitoring for survivor's supports, readiness, and adherence factors to minimize their impact on clinician dose determination in phase I of CDT.

\section{REFERENCES:}

1. Howlader N, Noone AM, Krapcho M, Neyman N, Aminou R, et al. SEER Cancer Statistics Review, 1975-2009 (Vintage 2009 Populations), National Cancer Institute. Bethesda, MD, 2012. Available at: http://seer.cancer.gov/csr/1975_2009_pops09/. Accessed March 10, 2013.

2. Tai YC, Domchek S, Parmigiani G, et al. Breast cancer risk among male BRCA1 and BRCA2 mutation carriers. J Natl Cancer Inst 2007;99:1811-4. [PMID: 18042939]

3. American Cancer Society. Breast Cancer. 2017. Available at: http://www.cancer.org/acs/groups/cid/documents/webcontent/003090-pdf Accessed September 6, 2017.

4. Fu M, Ridner S, Armer J. Post-breast cancer. Lymphedema: part 1. The Am J Nurs. 2009;109:48-54. [PMID: 19546644]

5. Cromwell KD, Chiang YJ, Armer J, Heppner PP, Mungovan K, et al. Is surviving enough? Coping and impact on activities of daily living among melanoma patients with lymphedema. Eur J Cancer Care. 2015;24:724-33. [PMID: 23752305]

6. Cheville AL, Mc Garvey CL, Petrek JA, Russo SA, Taylor ME, Thiadens SR. Lymphedema management. Semin Radiat Oncol, 2003;13:290-301. [PMID: 12903017]

7. Lasinski B, Thrift K, Squire D, Austin MK, Smith KM, et al. A systematic review of the evidence for complete decongestive therapy in the treatment of lymphedema from 2004 to 2011. PM \& R. 2012;4:580-601. [PMID: 2290313]

8. Foldi M, Foldi E. Lymphostatic Disease. In Foldi M, Foldi E. Textbook of Lymphology: For Physicians and Lymphedema Therapists, 2e. San Francisco, CA: Mosby; 2006, 224-319.

9. Yamamoto T, Todo, Y, Kaneuchi, M, Handa, Y, Watanabe, K, Yamamoto, R. Study of edema reduction patterns during the treatment phase of complex decongestive physiotherapy for extremity lymphedema. Lymphology. 2008;41:80-6.

10. Leduc $O$, Leduc $A$, Bourgeois $P$, Belgrado J. The physical treatment of upper limb edema. Cancer. 1998;83:2835-9. [PMID: 9874408]

11. McNeely ML, Magee D, Lees A, Bagnall K, Haykowsky M, Hanson J. The addition of manual lymph drainage to compression therapy for breast cancer related lymphedema: a randomized controlled trial. Breast Cancer Research and Treatment. 2004;86:95-106. [PMID: 15319562]

12. Dillman DA, Smyth JD, Christian LM. Internet, Mail, and Mixed Mode Surveys, the Tailored Design Method. New York: Wiley; 2009.

13. Salant P, Dillman D. How to Conduct Your Own Survey. New York: Wiley;1994.

14. Harris PA, Taylor R, Thielke R, Payne J, Gonzalez N, Conde JG. Research electronic data capture (REDCap) - A metadata-driven methodology and workflow process for providing translational research informatics support, J Biomed Inform. 2009;42:377-81. [PMID: 18929686]

15. Bernas $M$, Witte $M$, Kriederman $B$, Summers $P$, Witte $C$. Massage therapy in the treatment of lymphedema. Rationale, results, and applications. IEEE Engineering In Medicine And Biology Magazine. 2005;24:58-68. [PMID: 15825847]

16. Cheifetz O, Haley L. Breast Cancer Action. Management of secondary lymphedema related to breast cancer. Cancer Family Physician. 2010;56:1277-84. [PMID: 21375063]

17. Holtgrefe K. Twice-weekly complete decongestive physical therapy in the management of secondary lymphedema of the lower extremities. Physical Therapy. 2006;86:1128-36. [PMID: 16879046]

18. Sayko O, Pezzin L, Yen T, Nattinger A. Diagnosis and treatment of lymphedema following breast cancer: A population-based study. American Academy of Physical Medicine and Rehabilitation. 2013;5:915-23. [PMID: 23684778]

19. Hulett J, Armer J, Stewart B, Wanchai A. Perspectives of the breast cancer survivorship continuum: Diagnosis through 30 months post-treatment. J Pers Med. 2015;8:174-90. [PMID: 26030800]

20. Fenton N, Ferris N, Ko Z, Javalkar K, Hooper S. The relationship of health care transition readiness to diseaserelated characteristics, psychosocial factors, and health care outcomes: Preliminary findings in adolescents with chronic kidney disease. J Pediatr Rehabil Med. 2015;8:13-22. [PMID: 25737344]

(c) The Internet Journal of Allied Health Sciences and Practice, 2018 
21. Ridner S, Fu M, Wanchai A, Stewart B, Armer J, Cormier J. Self-Management of lymphedema: A systematic review of the literature from 2004 to 2011. Nursing Research. 2012;61:291-9. [PMID:22565103]

22. Brown J, Cheville A, Tchou J, Harris S, Schmitz K. Prescription and adherence to lymphedema self-care modalities among women with breast cancer-related lymphedema. Support Care Cancer. 2014;224:135-43. [PMID:24013569]

23. Nulty D. The adequacy of response rates to online and paper surveys: what can be done? Assessment \& Evaluation in Higher Education. 2008;33:301-14. 